\title{
Acute pancreatitis mimicking the electromechanical manifestations of ST-segment elevation myocardial infarction
}

\author{
Razi Khan MD¹, Hector Li Chang MD², Shahar Lavi MD
}

R Khan, HL Chang, S Lavi. Acute pancreatitis mimicking the electromechanical manifestations of ST-segment elevation myocardial infarction. Curr Res Cardiol 2014;1(2):117-119.

Mild electrocardiographic abnormalities are often observed in the setting of acute pancreatitis. The authors report a case involving a patient diag-

$\mathrm{T}$ he mechanism for electrocardiographic (ECG) abnormalities in the setting of acute pancreatitis remains unknown. We present a case involving a patient diagnosed with acute pancreatitis who had ECG findings suggestive of inferior ST-segment elevation myocardial infarction (STEMI) with concordant inferior wall motion abnormalities on echocardiography and patent coronary arteries.

\section{CASE PRESENTATION}

A 78-year-old man presented to a peripheral hospital after experiencing $2 \mathrm{~h}$ of nonexertional epigastric pain with progressive radiation to his chest. This was associated with nausea and diaphoresis, but no dyspnea, palpitations or presyncope. Electrocardiography was performed and revealed ST-segment elevation in the inferior leads suggestive of myocardial infarction (MI) (Figure 1A). The patient was transferred for cardiac catheterization. Physical examination of the patient revealed a

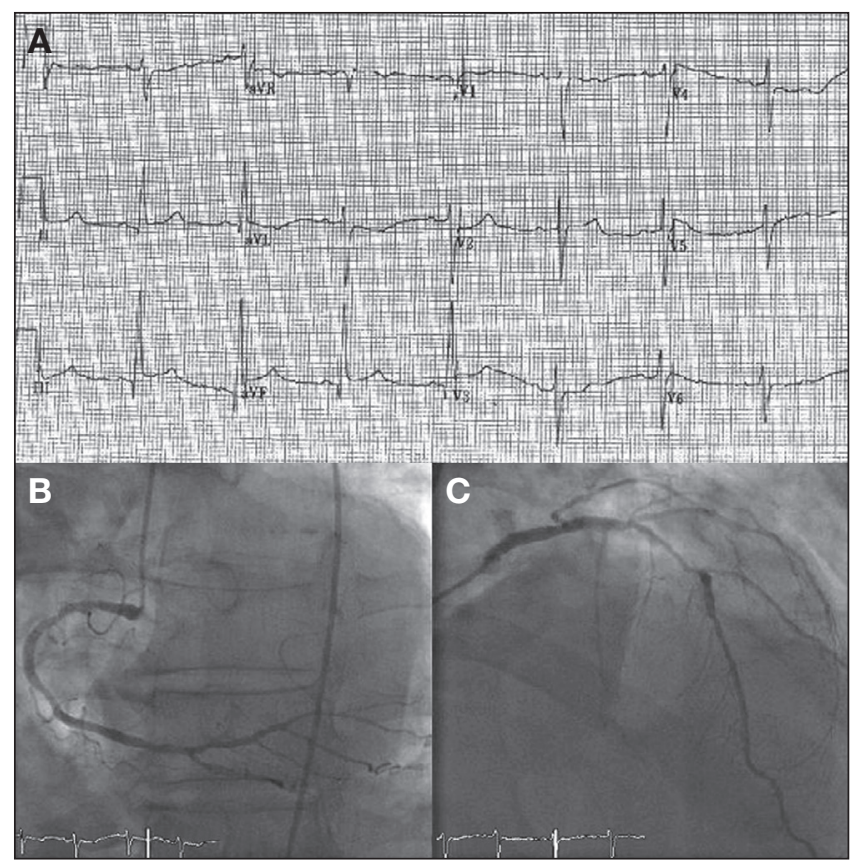

Figure 1) A Initial electrocardiogram depicting ST segment elevations in the inferior leads (II, III and aVF). Coronary angiography with absence of obstructive lesions in the right $(\mathrm{B})$ and left $(\mathrm{C})$ coronary arteries nosed with acute pancreatitis who had electrocardiographic findings of ST-elevation myocardial infarction with concordant echocardiographic findings of wall motion abnormalities, but patent coronary arteries and no evidence of myocardial injury on pathology.

Key Words: Acute pancreatitis; ECG abnormalities; ST elevation

diffusely distended abdomen with tympanic bowel sounds, but no signs of peritonitis. Although acute pancreatitis was suspected, the authors decided to proceed with coronary angiography to determine whether there was concomitant occlusion of a coronary artery. A minimal amount of contrast agent was used. Coronary angiography was only notable for calcified nonobstructive lesions in the left anterior descending artery (40\% to $50 \%)$ and right coronary artery (20\% to $30 \%)$ (Figures $1 \mathrm{~B}$ and $1 \mathrm{C}$ ). The left circumflex artery was small in size, supplied a small territory and had only mild narrowing. Echocardiography revealed hypokinesis of the inferior wall with mild reduction in right ventricular function (Figure 2). Subsequent electrocardiography demonstrated resolution of the ST-segment elevation (Figure 3).

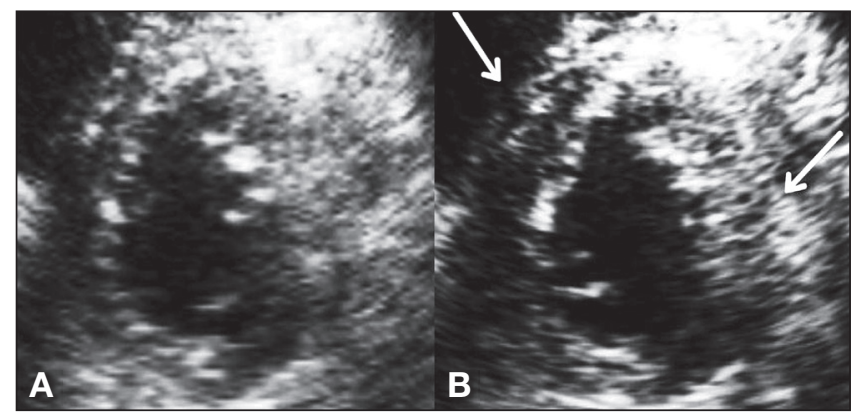

Figure 2) Parasternal short-axis echocardiographic views of the left ventricle during mid-diastole (A) and mid-systole (B). Evidence of myocardial thickening and motion is present for the anterior and lateral wall (arrows) but absent for the inferior wall

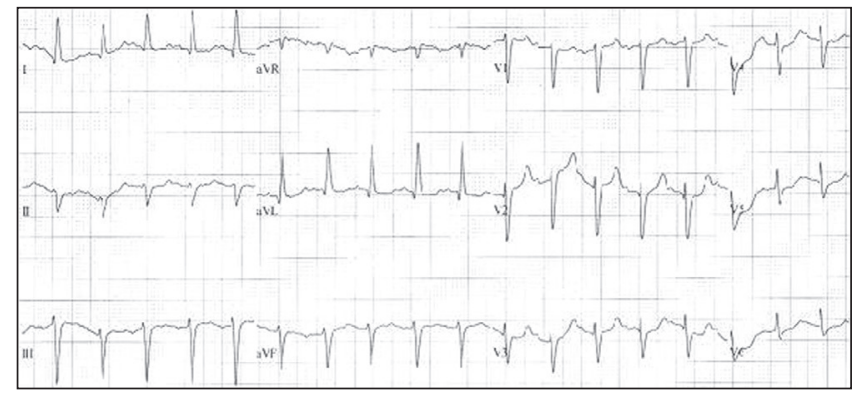

Figure 3) Subsequent electrocardiography reveals resolution of ST segment elevation

${ }^{1}$ Department of Internal Medicine, Division of Cardiology; ${ }^{2}$ Division of Pathology; University of Western Ontario, London, Ontario Correspondence: Dr Shahar Lavi, Division of Cardiology, The University of Western Ontario, 339 Winderemere Road, PO Box 5339, London,

Ontario N6A 5A5. Telephone 519-663-3611, fax 519-663-3117, e-mail shahar.lavi@lhsc.on.ca

\footnotetext{
OPEN ACCESS

This open-access article is distributed under the terms of the Creative Commons Attribution Non-Commercial License (CC BY-NC) (http:// ACCESS creativecommons.org/licenses/by-nc/4.0/), which permits reuse, distribution and reproduction of the article, provided that the original work is properly cited and the reuse is restricted to noncommercial purposes. For commercial reuse, contact support@pulsus.com
} 

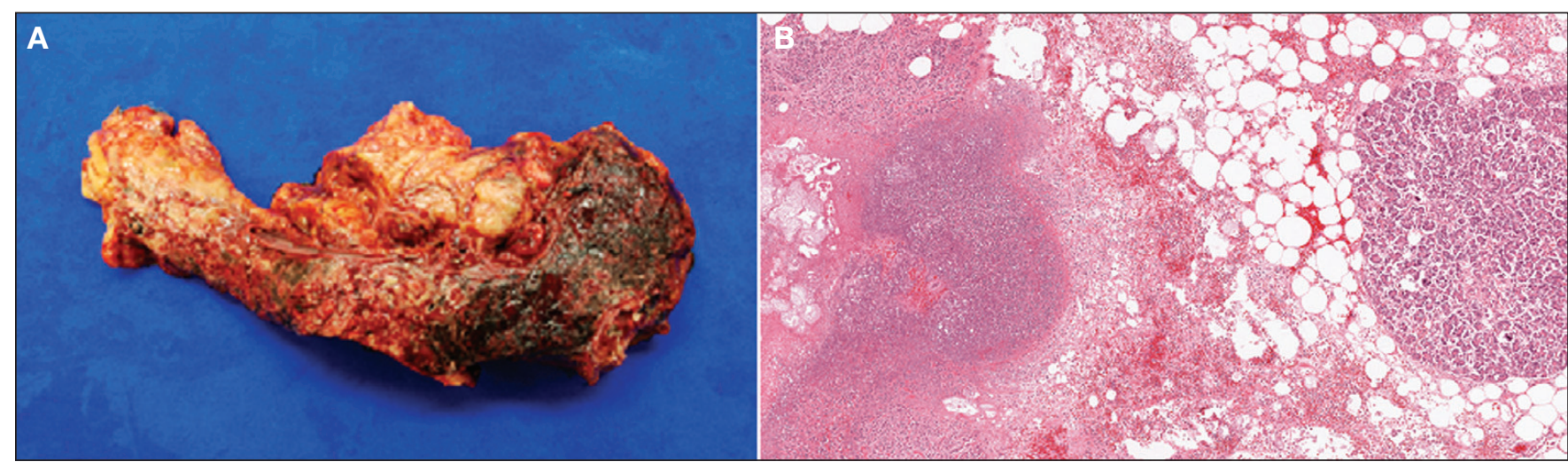

Figure 4) A Representative gross specimen of hemorrhagic pancreatitis. B Histological section showing necrosis and residual viable acini with surrounding areas of hemorrhage (original magnification $\times 2$ )

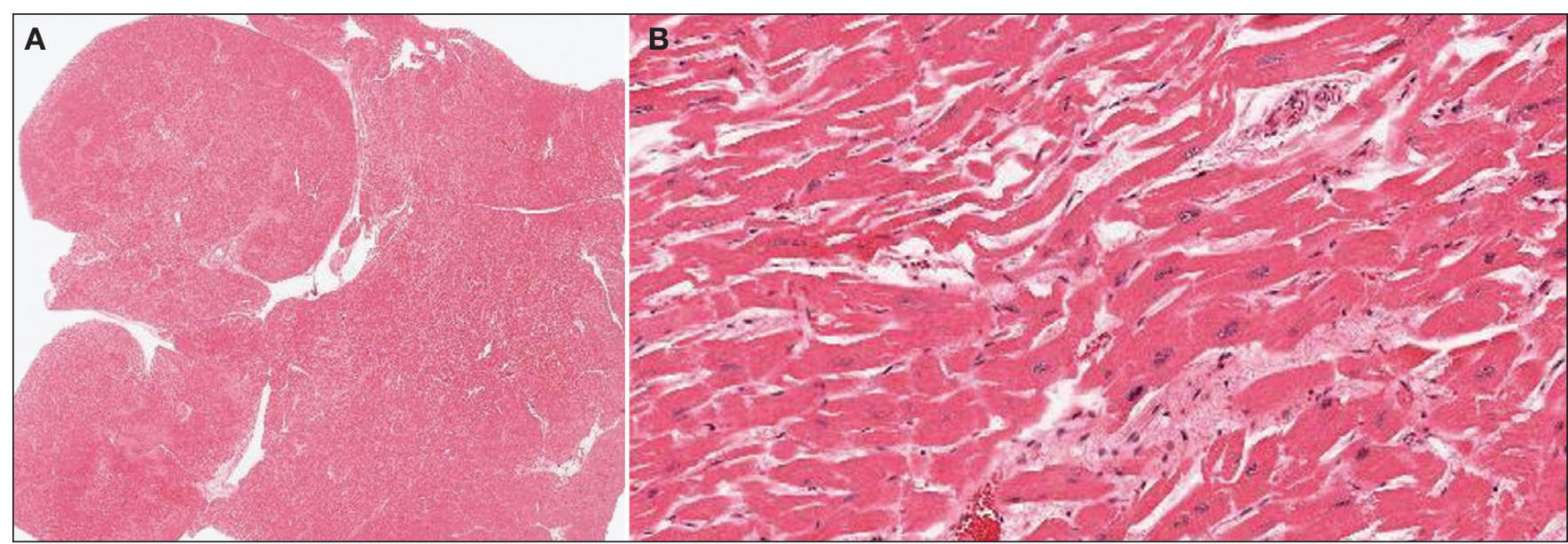

Figure 5) A Histological section of the inferior left ventricle (original magnification $\times 2$ ). Papillary muscles on left and epicardium on right. B Histological section of the myocardium. No evidence of coagulation necrosis, loss of nuclei or neutrophilic infiltration is present (original magnification $\times 20$ )

TABLE 1

\section{Cardiac markers}

\begin{tabular}{lccc}
\hline Marker & Day 1 & Day 2 & Day 3 \\
\hline Troponin T, $\mu \mathrm{g} / \mathrm{L}$ & $<0.01$ & $<0.01$ & 0.02 \\
Creatine kinase, U/L & 57 & 100 & 148 \\
\hline
\end{tabular}

Serum biochemisty demonstrated a lipase level of $>40,000 \mathrm{U} / \mathrm{L}$ and an amylase level of $1917 \mathrm{U} / \mathrm{L}$. Cardiac biomarkers, including troponin T, were and remained within normal range (Table 1). Computed tomography of the abdomen revealed evidence of necrotizing pancreatitis. No primary cause for his pancreatitis was determined.

One day after admission, the patient began complaining of increasing abdominal pain. He then developed acute respiratory distress syndrome and required mechanical ventilation. Subsequently, there was deterioration in his hemodynamic status and renal function. The patient died due to acute respiratory distress syndrome and vasodilatory shock, despite intensive support, including administration of vasopressors, $46 \mathrm{~h}$ after the electrocardiography mimicking STEMI was performed. An autopsy confirmed the presence of necrotizing pancreatitis (Figure 4). Although nonobstructive calcified coronary lesions were noted, there was no evidence of a new thrombotic occlusion or myocardial necrosis on histology (Figure 5), confirming that the ST-segment elevation on electrocardiography did not reflect MI.

\section{DISCUSSION}

To our knowledge, the present case is the first involving a patient with concurrent ECG and echocardiographic abnormalities suggestive of ischemia, in which the absence of MI had been confirmed on pathology. The present case helps to substantiate the fact that acute pancreatitis can mimic both electrical and mechanical manifestations of acute MI without occlusion of coronary arteries. ECG abnormalities have been frequently documented in patients with acute pancreatitis. The most common ECG changes consist of nonspecific repolarization abnormalities (1). Although documented, ST-segment elevation is rare (1-4). The presence of associated wall motion abnormalities on echocardiography is even more infrequent $(2-4)$. The cause of both ECG and echocardiographic abnormalities observed with pancreatitis remains unknown.

Coronary vasospasm or transient occlusion due to thrombus could cause both ST-segment elevation on electrocardiography, and wall motion abnormalities on the echocardiogram performed on our patient. In fact, Phadke et al (5) reported findings of thrombus in the left anterior descending artery in a patient who presented with symptoms and findings of MI following acute pancreatitis (5). However, in such a case, even if there is no evidence of clot at the time of angiography, there is likely to be evidence of myocardial injury and elevated cardiac marker levels. Vasospasm has been documented to be the most common cause of ST-segment elevation in the absence of epicardial disease (6). Pancreatitis may induce vasospasm by causing endothelial dysfunction of the coronary arteries. However, there was no evidence of vasospasm noted on angiography. In addition, the presence of coronary thombosis was also not visualized on angiography, nor was it described on cardiac pathology.

Takotsubo cardiomyopathy - a frequent cause of wall motion abnormalities described in the absence of obstructive coronary disease 
- has been associated with critical illness and may have been partially responsible for the electromechanical cardiac abnormalities (7). However, there was no evidence of typical apical ballooning observed with Takotsubo cardiomyopathy. There was also an absence of basal sparing of inferior wall motion abnormalities, also suggesting an alternative diagnosis. As with Takotsubo cardiomyopathy in the critically ill, pancreatitis may induce myocardial dysfunction through the release of cardiotoxic inflammatory mediators, resulting in wall motion abnormalities (8).

Electrolyte abnormalities, found in up to $50 \%$ of patients with pancreatitis (9), have been previously noted to cause ECG changes that may be consistent with MI. However, ECG abnormalities have been documented in individuals with normal electrolyte panels, as was the case with our patient (3). In addition, the presence of concurrent wall motion abnormalities would not be explained. The cardiobiliary

\section{REFERENCES}

1. Tejada J, Hernandez F, Chimeno J, Alonso M, Martin R, Bastante T. Acute pancreatitis mimicking acute inferior myocardial infarction. Angiology 2008;59:365-7.

2. Patel J, Movahed A, Reeves W. Electrocardiographic and segmental wall motion abnormalities in pancreatitis mimicking myocardial infarction. Clin Cardiol 1994;17:505-9.

3. Khairy P, Marsolais P. Pancreatitis with electrocardiographic changes mimicking acute myocardial infarction. Can J Gastroenterol 2001;15:522-26.

4. Ro T, Lang R, Ward R. Acute pancreatitis mimicking myocardial infarction: Evaluation with myocardial contrast echocardiography. J Am Society Echocardiography 2004;17:387-90.

5. Phadke MS, Punjabi P, Sharma S, Kide S, Nawale J, Chaurasia A. Acute pancreatitis complicated by ST-elevation myocardial infarction. J Emerg Med 2013;44:932-5. reflex associated with increased vagal tone has been suggested to be a mechanism for ECG changes in pancreatitis; however, the presence of ST-segment elevation would be unexpected (3). As with wall motion abnormalities, coronary vasospasm or transient coronary thrombus may have been the most likely causes of dynamic ST-segment elevation observed in this patient.

\section{CONCLUSION}

Despite the findings of ST-segment elevation and concurrent wall motion abnormalities in the present case, pathology noted the absence of $\mathrm{MI}$ as a cause for the ECG and echocardiographic abnormalities. Therefore, the present report confirms that acute pancreatitis alone can mimic the electromechanical manifestations of STEMI without obvious obstruction of the coronary arteries. The etiology of these cardiac-related changes is unknown and remains speculative.

6. Da Costa A, Isaaz K, Faure E, Mourot S, Cerisier A, Lamaud M. Clinical characteristics, aetiological factors and long-term prognosis of myocardial infarction with an absolutely normal coronary angiogram: A 3-year follow-up study of 91 patients. Eur Heart J 2001;22:1459-65.

7. Chockalingam A, Mehra A, Dorairajan S, Dellsperger KC. Acute left ventricular dysfunction in the critically ill. Chest 2010;138:198-207.

8. Waldo SW, Rosario HS, Penn AH, Schmid-Schönbein GW. Pancreatic digestive enzymes are potent generators of mediators for leukocyte activation and mortality. Shock 2003;20:138-43.

9. Pezzilli R, Barakat B, Billi P, Bertaccini B. Electrocardiographic abnormalities in acute pancreatitis. Eur J Emerg Med 1999;6:27-9. 